\title{
IS SGR 0418+5729 INDEED A WANING MAGNETAR?
}

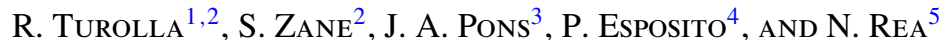 \\ ${ }^{1}$ Department of Physics, University of Padova, Via Marzolo 8, I-35131 Padova, Italy \\ ${ }^{2}$ Mullard Space Science Laboratory, University College London, Holmbury St. Mary, Dorking, Surrey, RH5 6NT, UK \\ ${ }^{3}$ Department de Fisica Aplicada, Universitat d'Alacant, Ap. Correus 99, 03080 Alacant, Spain \\ ${ }^{4}$ INAF-Astronomical Observatory of Cagliari, Località Poggio dei Pini, Strada 54, I-09012 Capoterra, Italy \\ ${ }^{5}$ Institut de Ciències de l'Espai (CSIC-IEEC), Campus UAB, Facultat de Ciències, Torre C5-parell, E-08193 Barcelona, Spain \\ Received 2011 June 1; accepted 2011 July 27; published 2011 October 4
}

\begin{abstract}
SGR $0418+5729$ is a transient soft gamma-ray repeater which underwent a major outburst in 2009 June, during which the emission of short bursts was observed. Its properties appeared quite typical of other sources of the same class until long-term X-ray monitoring failed to detect any period derivative. The present upper limit on $\dot{P}$ implies that the surface dipole field is $B_{p} \lesssim 7.5 \times 10^{12} \mathrm{G}$, well below those measured in other soft gamma-ray repeaters (SGRs) and in the Anomalous X-ray Pulsars (AXPs), a group of similar sources. Both SGRs and AXPs are currently believed to be powered by ultra-magnetized neutron stars (magnetars, $B_{p} \approx 10^{14}-10^{15} \mathrm{G}$ ). SGR $0418+5729$ hardly seems to fit in such a picture. We show that the magneto-rotational properties of SGR $0418+5729$ can be reproduced if this is an aged magnetar, $\approx 1$ Myr old, which experienced substantial field decay. The large initial toroidal component of the internal field required to match the observed properties of SGR 0418+5729 ensures that crustal fractures, and hence bursting activity, can still occur at the present time. The thermal spectrum observed during the outburst decay is compatible with the predictions of a resonant Compton scattering model (as in other SGRs/AXPs) if the field is low and the magnetospheric twist is moderate.
\end{abstract}

Key words: pulsars: individual (SGR 0418+5729) - stars: magnetic field - stars: neutron

Online-only material: color figure

\section{INTRODUCTION}

Soft gamma repeaters (SGRs) and Anomalous X-ray Pulsars (AXPs) form a small, but rapidly expanding, class of isolated neutron star (NS) sources characterized by the emission of short $(\approx 0.1 \mathrm{~s})$, energetic $\left(\approx 10^{40} \mathrm{erg} \mathrm{s}^{-1}\right)$ bursts of X-rays. Their persistent luminosity $\left(L_{X} \approx 10^{32}-10^{36} \mathrm{erg} \mathrm{s}^{-1}\right.$ in the $\sim 0.5-10 \mathrm{keV}$ range) is often variable, with flux enhancements up to several hundreds during the outburst phases of transient sources (e.g., Rea \& Esposito 2011). SGRs and AXPs share similar timing properties, with periods in a narrow range $(P \sim$ $2-12 \mathrm{~s}$ ), and large period derivatives ( $\dot{P} \approx 10^{-13}$ to $10^{-10} \mathrm{~s} \mathrm{~s}^{-1}$ ). In most of these sources the X-ray luminosity exceeds the rate of rotational energy losses $\left(\dot{E} \approx 10^{32}-10^{35} \mathrm{erg} \mathrm{s}^{-1}\right.$ ), and highly variable radio activity has been detected in three objects. $\mathrm{X}$-ray spectra are often characterized by a blackbody (BB; $k T \approx 0.5 \mathrm{keV}$ ) plus a high-energy power-law (PL; photon index $\Gamma \approx 1.5-3$ ) component (e.g., Rea et al. 2008), or by two thermal components (e.g., Gotthelf \& Halpern 2007; Tiengo et al. 2008). Both the spectral parameters and pulse profiles appear to vary with the source flux (see, e.g., Woods \& Thompson 2006; Mereghetti 2008; Rea \& Esposito 2011, for reviews on SGR/AXP properties). ${ }^{6}$

The large values of the magnetic field derived from the standard dipole formula $\left(5 \times 10^{13} \mathrm{G} \lesssim B_{p} \lesssim 10^{15} \mathrm{G}\right)$, the lack of detected stellar companions, and their large $\mathrm{X}$-ray output as compared to $\dot{E}$ led to the suggestion that SGRs and AXPs are powered by a young (age $\approx 10^{3}-10^{4} \mathrm{yr}$ ), ultra-magnetized NS, or magnetar (Duncan \& Thompson 1992; Thompson \& Duncan 1993). Indeed, the magnetar scenario has been largely successful in explaining many of the observed properties of SGRs/AXPs,

\footnotetext{
6 See also http://www.physics.mcgill.ca/ pulsar/magnetar/main.html for an updated catalog of SGRs/AXPs.
}

including those of the bursts (Thompson \& Duncan 1995) and of the persistent emission (the twisted magnetosphere model, Thompson et al. 2002; Zane et al. 2009; Albano et al. 2010, and references therein).

Despite that SGRs and AXPs are far from being a homogeneous class, in particular the inferred surface dipolar field spans nearly two orders of magnitude, their observational behavior is now commonly associated with that of (active) magnetars, to the point that often the terms SGR/AXP and magnetar are used as synonyms. This, actually, reflects the original definition of a magnetar as an NS which is powered by its (large) magnetic field (Thompson \& Duncan 1993). In this respect, a super-strong magnetic field is not per se a sufficient condition for triggering SGR/AXP-like activity, as testified by the existence of NS sources, for instance most of the so-called High-B radio pulsars (HBPSRs; e.g., Kaspi 2010), and possibly some of the thermally emitting isolated NSs (XDINSs; e.g., Turolla 2009), with surface magnetic fields comparable to those of SGRs/AXPs but having substantially different properties and not showing any bursting/outbursting activity over the $\sim 10-20 \mathrm{yr}$ time span during which they were observed.

Over the last few years, the family of the magnetar candidates grew with the addition of several new objects, most of them transient. X-ray bursting activity or peculiar radio emission similar to that of SGRs /AXPs was detected in allegedly rotationpowered pulsars, such as PSR J1846-0258 (Gavriil et al. 2008; Kumar \& Safi-Harb 2008) and PSR 1622-4950 (Levin et al. 2010). This suggests that magnetic energy substantially contributes to their emission at certain stages. The magnetic field inferred from their rotation properties $\left(B_{p} \sim(0.5-3) \times 10^{14} \mathrm{G}\right)$ is, in fact, close to that of SGRs/AXPs, contributing to the widespread belief that magnetar-like activity has to be associated with super-strong magnetic fields, typically higher than the quantum field $B_{Q} \simeq 4.4 \times 10^{13} \mathrm{G}$. 
In this respect, the recent discovery of a low-field SGR, SGR 0418+5729 (Rea et al. 2010), came as a surprise. SGR 0418+5729 was observed for the first time in 2009 June when it entered an outburst state during which X-ray bursts were detected (van der Horst et al. 2010). The enhanced flux level allowed for a measure of the source periodicity, $P \sim 9.1 \mathrm{~s}$, but, despite that the source was then monitored in X-rays for $\sim 500$ days, no significant evidence for a period derivative was found (Esposito et al. 2010; Rea et al. 2010, and references therein). The published upper limit is $\dot{P} \lesssim 6 \times 10^{-15} \mathrm{~s} \mathrm{~s}^{-1}$, leading to an inferred dipole field $B_{p} \lesssim 7.5 \times 10^{12} \mathrm{G}$.

The very low magnetic field of SGR $0418+5729$ (when compared with other SGRs/AXPs) raises a number of questions as to if, and how, the observed phenomenology of this source can be accommodated within the magnetar picture. A crucial point is whether an NS with a surface field well below $10^{13} \mathrm{G}$ can harbor an internal toroidal magnetic field strong enough to produce crustal displacements, which are believed to be responsible for the bursting/outbursting episodes in SGRs/AXPs (Thompson \& Duncan 1995; Thompson et al. 2002; Beloborodov 2009). In this paper, we address this issue and discuss both the spectral and timing properties of SGR $0418+5729$ in the framework of an aging magnetar.

\section{SGR 0418+5729 AS AN OLD MAGNETAR}

The suggestion that SGR 0418+5729 may be an aged magnetar was already put forward by Rea et al. (2010; see also Esposito et al. 2010) on the basis of the low persistent luminosity (most likely well below that observed during the outburst decay, $\left.L_{X} \sim 6 \times 10^{31}(D / 2 \mathrm{kpc}) \mathrm{erg} \mathrm{s}^{-1}\right)$, weak bursting activity (only two faint bursts were recorded; van der Horst et al. 2010), and large spin-down age $\left(t_{c} \gtrsim 24 \mathrm{Myr}\right)$. Rea et al. (2010) estimated that an internal toroidal field $B_{\text {tor }} \approx 5 \times 10^{14} \mathrm{G}$ is required to power the persistent emission over a source lifetime $\sim t_{c}$, and concluded that $B_{\text {tor }}$ can be still large enough to overcome the crustal yield.

The main appeal of the "old magnetar" scenario is that it can offer an interpretation of the observed properties of SGR 0418+5729 within an already established framework, validating the magnetar model also for (surface) field strengths quite far away from those of canonical SGRs/AXPs. However intriguing, the considerations presented by Rea et al. (2010) necessary rely on quite crude estimates. A more thorough investigation on the behavior of evolved magnetars, in which a substantial decay of the magnetic field occurred, is definitely in order before claiming that SGR $0418+5729$ is indeed powered by the last hiccups of a once-ultra-magnetized NS.

In the following we focus on three points which we deem central in order to test the "old magnetar" hypothesis. First, assuming that SGR $0418+5729$ was born with a magnetarlike surface field, $B_{p}$ must have decayed by a factor $\approx 100$ to match the current upper limit. Roughly the same reduction is expected in the internal field. Although the latter can initially be $\approx 10-100$ times higher than $B_{p}$ (at least locally), one may wonder if at late times internal magnetic stresses are still strong enough to crack the crust. A second and related question is if realistic models of field decay in magnetars can account for the observed rotational properties (period and period derivative) of SGR $0418+5729$. This also has direct bearing on the true age of the source, which is most probably much younger than the characteristic age, estimated assuming a non-decaying field. A final point concerns the persistent emission of SGR 0418+5729. XMM-Newton observations show evidence for a two-component thermal spectrum (similar to those observed in other transient magnetars), although the presence of a non-thermal tail cannot be excluded (Esposito et al. 2010, 2011; see Section 2.4). In the magnetar model, spectra are expected to exhibit a PL tail that originates from resonant scattering in a twisted magnetosphere (Thompson et al. 2002). However, no calculations have been performed yet in the range of surface fields implied by SGR 0418+5729.

\subsection{Magneto-rotational Evolution}

A major issue in establishing the magnetic evolution of NSs (and of magnetars in particular) is that observations place very little, if any, constraint on the structure and strength of the internal magnetic field. While there are several indications that the large-scale, external field can be reasonably assumed to be dipolar, with a moderate amount of twist in magnetars, the different mechanisms proposed for the generation of the internal field in the earlier phases of the NS life (differential rotation, dynamo, magneto-rotational instability) most likely give rise to both toroidal and poloidal components (e.g., Geppert et al. 2004, 2006 and references therein). The presence of a toroidal field, roughly in equipartition with the poloidal one, is also required by general stability arguments (e.g., Braithwaite \& Spruit 2006 and references therein). A further complication comes from the present poor knowledge of where the internal field resides. The field can either permeate the entire star ("core" fields), or be mostly confined in the crust ("crustal" fields), depending on where its supporting (super) currents are located. The highly anisotropic surface temperature distribution required in some XDINSs has been taken as observational evidence in favor of a complex field geometry in the external layers (crust, envelope, atmosphere) of NSs, either in the form of strong crustal toroidal fields, multipolar poloidal components, or both (Geppert et al. 2006; Pérez-Azorín et al. 2006; Zane \& Turolla 2006).

The more general configuration for the internal field in a NS will then be that produced by the superposition of current systems in the core and the crust. As stressed by Pons \& Geppert (2007), the relative contribution of the core/crustal fields is likely different in different types of NSs. In old radio pulsars, where no field decay is observed, the long-lived core component may dominate, while a sizable, more volatile crustal field is probably present in magnetars, for which substantial field decay over a timescale $\approx 10^{3}-10^{5} \mathrm{yr}$ is expected (e.g., Goldreich \& Reisenegger 1992).

A particularly important result (Glampedakis et al. 2011) is the lesser role that ambipolar diffusion plays in magnetar cores (after crystallization, the absence of convective motions already quenched ambipolar diffusion in the crust) on their active lifetimes, contradicting an assumption often made in the modeling of the flaring activity. Therefore, if the decay/ evolution of the magnetic field is indeed the cause of magnetar activity, it is likely to take place outside the core and will be governed by Hall/Ohmic diffusion in the stellar crust. The relative importance of these two mechanism is strongly density and temperature dependent. Thus, any self-consistent study of the magnetic field evolution must be coupled to a detailed modeling of the NS thermal evolution, and vice versa. Other mechanisms, e.g., flux expulsion from the superconducting core, due to the interaction between neutron vortices and magnetic flux tubes, are highly uncertain and very difficult to model. For these reasons, recent investigations of the magnetic field evolution in magnetars focused only on the crustal component of the field. 

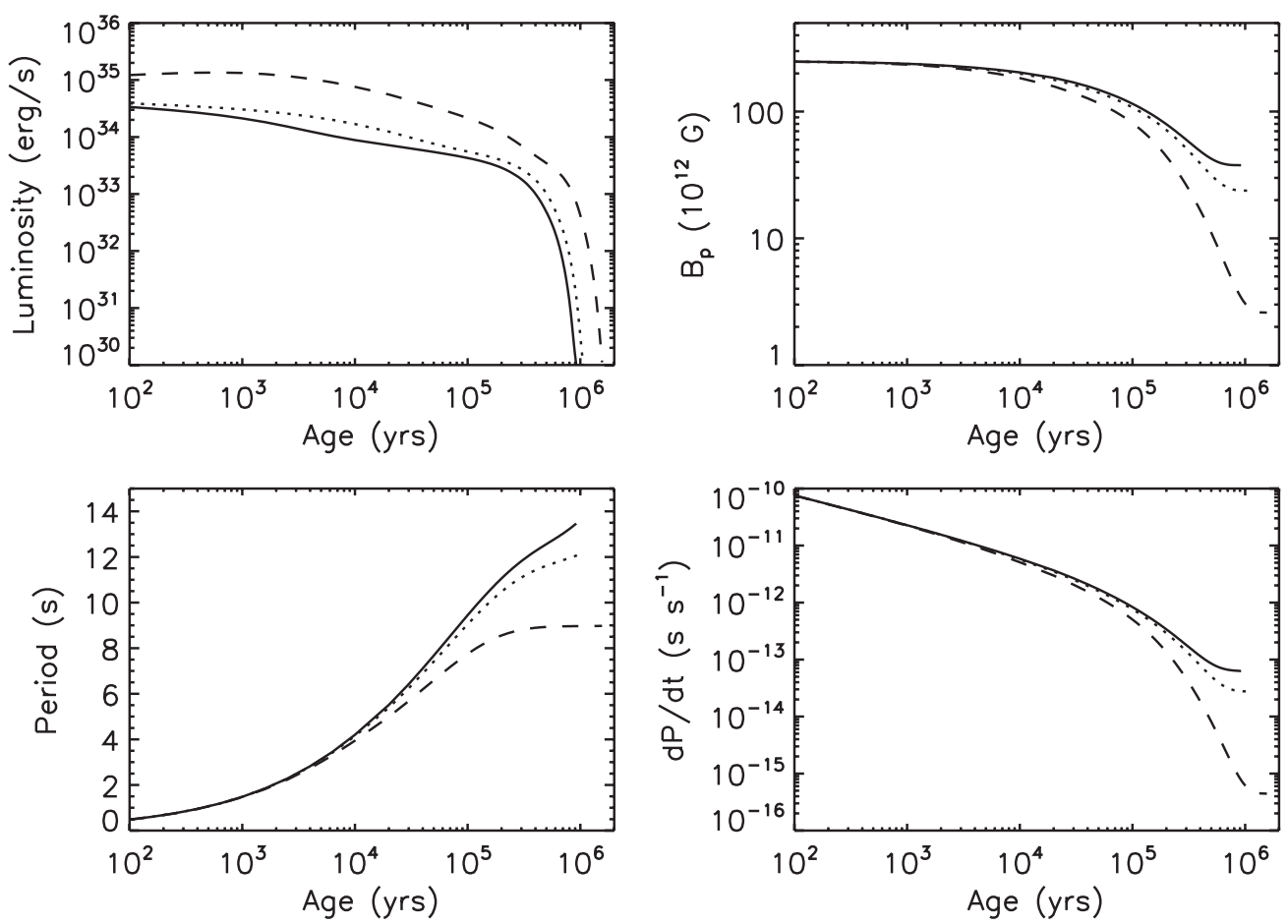

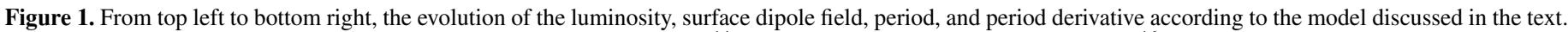
The three cases refer to $B_{\mathrm{tor}}(t=0)=0$ (solid lines), $B_{\mathrm{tor}}(t=0)=4 \times 10^{14} \mathrm{G}($ dotted lines $)$, and $B_{\mathrm{tor}}(t=0)=4 \times 10^{16} \mathrm{G}(\mathrm{dashed}$ lines $)$.

The first attempts in this direction used a split approach. Pons \& Geppert (2007) studied the evolution of the field by solving the complete induction equation in an isothermal crust, but assuming a prescribed time dependence for the temperature. They found that crustal magnetic fields in NSs suffer significant decay during the first $\approx 10^{6} \mathrm{yr}$ and that the Hall drift, although inherently conservative (i.e., alone it cannot dissipate magnetic energy), plays an important role since it may reorganize the field from the larger to the smaller (spatial) scales where Ohmic dissipation proceeds faster.

The cooling of magnetized NSs with field decay was investigated by Aguilera et al. (2008) by adopting a simple, analytical law for the time variation of the field which incorporates the main features of the Ohmic and Hall terms in the induction equation. The fully coupled magneto-thermal evolution of an NS was finally addressed by Pons et al. (2009), including all realistic microphysics. However, owing to numerical difficulties in treating the Hall term, their models include only Ohmic diffusion. This can be a limitation because, as they note, the Hall drift likely drastically affects the very early evolution of ultra-magnetized NSs with surface field values of $B_{p} \gtrsim 10^{15} \mathrm{G}$, and also that of "normal" NSs at late times ( $\left.\gtrsim 10^{6} \mathrm{yr}\right)$, when the temperature in the crust has dropped. On the other hand, for initial values of $B_{p} \lesssim 10^{15} \mathrm{G}$, still well within the magnetar range, the effect of the Hall drift is expected to introduce at most quantitative changes (a somewhat faster dissipation) with respect to the purely Ohmic picture.

\subsection{The Case of SGR $0418+5729$}

To explore if, and to which extent, the magneto-thermal evolution of (initially) highly magnetic NSs can lead to objects with properties compatible with those of SGR $0418+5729$, we performed some runs using the code of Pons et al. (2009). We refer to Section 2 in Pons et al. (2009) and Section 4 of Aguilera et al. (2008) for all details about the code and the microphysical input. We evolved a $1.4 M_{\odot}$ NS assuming the minimal cooling scenario (Page et al. 2004), with no exotic phases or fast neutrino cooling processes, but including enhanced neutrino emission from the breaking and formation of neutron Cooper pairs in the NS core, as recent observations of the Cassiopeia A supernova remnant seem to require (Page et al. 2011; Shternin et al. 2011). The initial period was fixed at $10 \mathrm{~ms}$ and the initial dipole field to $B_{p}=2.5 \times 10^{14} \mathrm{G}$. Note that the internal poloidal field is actually higher, with a maximum value $B_{\text {pol }}(t=0) \simeq 2.5 \times 10^{15} \mathrm{G}$ in the inner crust.

We considered three models with different values of the (maximum) internal toroidal field, $B_{\text {tor }}(t=0)=0,4 \times 10^{14}$ and $4 \times 10^{16} \mathrm{G}$, which turns out to be the crucial parameter, as shown in Figure 1. The four panels illustrate the evolution of luminosity, dipole field $B_{p}$, period $P$, and period derivative $\dot{P}$. Indeed the properties of SGR $0418+5729$ are recovered in the case of $B_{\text {tor }}(t=0)=4 \times 10^{16} \mathrm{G}$ and age $\sim 1.5 \times 10^{6} \mathrm{yr}$. The main conclusions can be summarized as follows.

1. The low quiescent luminosity is easily explained considering that the object is relatively old: even an NS born as a bright, hot magnetar becomes cool and dim at this age.

2. On the other hand, the observed period constrains the dipolar field: the initial dipole field cannot be much higher than that the one considered here in order to prevent the star from spinning down too fast and reaching periods longer than that observed at present. Obviously there are other large uncertainties, such as the angle between rotation and magnetic axis, that may reduce the period (we assumed an orthogonal rotator here).

3. Although the components of the initial internal field $B_{\text {tor }}(t=0)$ can be varied to some extent, a quite large value is required. A large toroidal field, in fact, implies strong currents, which, in turn, produce more heating and higher temperatures. This drives a faster global field decay, 
which makes it possible to match the observed upper limit on $\dot{P}$ and $B_{p}$.

We stress that, while there are no stringent arguments against such large internal fields in the NS crust, their real occurrence in magnetars is an open issue. A possibility is that if the Hall drift becomes very important and it results in much faster dissipation, then one can obtain the same results starting with lower initial toroidal fields. Finally, we note that the Hall term is bound to become important again for objects like SGR 0418+5729 at late stages ( $\gtrsim 1 \mathrm{Myr}$ ) as the star cools down and the conductivity increases by several orders of magnitude. No calculations are available in this regime but the possible occurrence of a second "Hall-active" phase could lead to enhanced bursting activity and rapid field decay. This may be an indication that the estimate of the bursting rate in Perna \& Pons (2011) is a lower limit.

\subsection{Occurrence of Bursts}

Very recently Perna \& Pons (2011) used the magnetic evolution code of Pons \& Geppert (2007) together with the cooling models by Pons et al. (2009) to compute the magnetic stress acting on the NS crust at different times. Their baseline model has $B_{p}(t=0)=8 \times 10^{14} \mathrm{G}$ and $B_{\text {tor }}(t=0)=10^{15} \mathrm{G}$. They found that the occurrence of crustal fractures (and hence of bursts) is not restricted to the early NS life, during which the surface field is ultra-strong, but can extend to late phases (age $\approx 10^{5}-10^{6}$ yr; see their Figure 2 ). Both the energetics and the recurrence time of the events evolve as the star ages. For "old" magnetars about $50 \%$ crustal fractures release $\approx 10^{41} \mathrm{erg}$ and the waiting time between two successive events is $\approx 1-10 \mathrm{yr}$. They also made a longer run with a model with $B_{p}(t=0)=$ $2 \times 10^{14} \mathrm{G}$ and $B_{\text {tor }}(t=0)=10^{15} \mathrm{G}$, for which the event rate is about a factor of 10 smaller.

The model we considered in Section 2.1 as representative of SGR $0418+5729$ has $B_{p}(t=0)$, very close to this latter configuration, while $B_{\text {tor }}(t=0)$ is larger. The present (maximum) value of the internal toroidal field is $\sim 9 \times 10^{14} \mathrm{G}$ and, although we did not perform any detailed simulations, we argue that the bursting rate of our model, at its present age, is similar to the second model of Perna \& Pons (2011), because the internal configuration of the magnetic field is similar. It is important to note that, despite the much larger initial toroidal fields of the model presented in this paper, this leads to faster decay and therefore similar values are reached when the NS is a million years old. Comparing both models at the estimated age of $1.5 \mathrm{Myr}$, the internal toroidal field of the model presented in this paper is only two times larger than that discussed in Perna \& Pons (2011), and we estimate that the typical lapse time between events for an object like SGR $0418+5729$ is $\sim 20-50$ years.

\subsection{Persistent Emission}

In order to investigate the spectral properties of the persistent emission from SGR $0418+5729$ and its time evolution, we analyzed eight Swift X-Ray Telescope $(\mathrm{XRT})^{7}$ and one XMMNewton/EPIC spectra (see Table S1 of Rea et al. 2010 for more details). Preliminary results for some of these data sets were already reported in Esposito et al. (2010, 2011). Spectra for all the epochs were fitted simultaneously using XSPEC v.12.6, with the value of the column density $N_{\mathrm{H}}$ tied within the different observations. Several one- and two-component models were

\footnotetext{
7 Each Swift data set contains several individual observations in order to obtain good enough statistics.
}

tried, including a single blackbody, a one-dimensional (RCS; Rea et al. 2008) and a three-dimensional (NTZ; Zane et al. 2009) resonant scattering model, a double blackbody and a blackbody plus a PL. All single component models give rather poor fits. While for the RCS and NTZ models the residuals and the $\chi^{2}$ values were not acceptable, a single $\mathrm{BB}$ decomposition properly reproduces all the data but the XMM-Newton spectrum, which is the only one responsible for the relatively large $\chi^{2}$ of the simultaneous fit. Since the highest-quality available spectrum argues against the source having (at least at the early stage of the outburst) a single thermal spectrum, we decided to add a second component to the multi-instrument fit.

$\mathrm{A} \mathrm{BB}+\mathrm{BB}$ and a $\mathrm{BB}+\mathrm{PL}$ model with all parameters free (except $N_{\mathrm{H}}$, see above) provide acceptable fits of comparable quality $\left(\chi_{\text {red }}^{2}=1.15\right.$ for 601 degrees of freedom (dof) and $\chi_{\text {red }}^{2}=1.12$ for 601 dof, respectively). However, we stress again that both these spectral representations contain a large number (32 in total) of free parameters, and those associated with the second component are not required by the seven Swift observations but only by the XMM-Newton one. Moreover, despite that on a statistical ground there is no reason to prefer the $\mathrm{BB}+\mathrm{BB}$ over the $\mathrm{BB}+\mathrm{PL}$ model, we note that in the latter: (1) the spectral index $\Gamma$ changes dramatically and in a totally erratic way from one observation to another and (2) $\Gamma$ can be as large as $\sim 6$, arguing against a PL as a physically motivated representation of the second spectral component. On the other hand, the values of the spectral parameters in the $\mathrm{BB}+\mathrm{BB}$ model appear to be reasonable and their time evolution is monotonic (see below).

Since in the BB+BB best-fit model the temperature and normalization of the colder BB component appear not to vary sensibly in time (again, possibly because they are poorly constrained by the Swift observations), we performed a fit with these two parameters tied across the various data sets. This resulted in a similarly good fit ( $\chi_{\text {red }}^{2}=1.18$ for 617 dof) and has the advantage of containing 16 degrees of freedom less. In the case of the BB+PL model the goodness of the fit worsens considerably $\left(\chi_{\text {red }}^{2}=1.42\right.$ for 617 dof $)$ by requiring that the parameters of the (single) BB are the same at the different epochs.

For these reasons, in the following we take the $\mathrm{BB}+\mathrm{BB}$ model (with the colder BB constant in time) as the most likely representation of the data, and discuss the ensuing implications in the framework of an evolved magnetar. It is worth mentioning that the failure of the resonant scattering models to fit the data may be due to the fact that both RCS and NTZ were originally developed for much higher fields than that likely present in SGR 0418+5729 (the NTZ version used here assumes $B_{p}=$ $\left.10^{14} \mathrm{G}\right)$. A more detailed spectral analysis will be the subject of a forthcoming paper (N. Rea et al. 2011, in preparation).

The picture which emerges from the spectral analysis is that of thermal emission from two regions on the star surface, a cold one, with more or less constant size and temperature $\left(R_{c} \sim 0.75 \mathrm{~km}\right.$ for a fiducial distance $D=2 \mathrm{kpc}$ and $T_{c} \sim 0.31 \mathrm{keV}$ ), and a hot one, which shrinks during the outburst decay. The evolution of the temperature and size of the two components is shown in Figure 2. The temperature of the hot region is more or less constant at $k T_{h} \sim 0.93$ and its area changes from $\sim 0.2$ to $\sim 0.03$ times that of the cold region $\left(R_{h} \sim 0.15-0.30 \mathrm{~km}\right.$, again for $\left.D=2 \mathrm{kpc}\right)$. The overall behavior is quite reminiscent of those seen in other transient magnetar sources, notably the AXPs XTE J1810-197 and CXOU J164710.2-455216 (e.g., Albano et al. 2010 and references therein). 


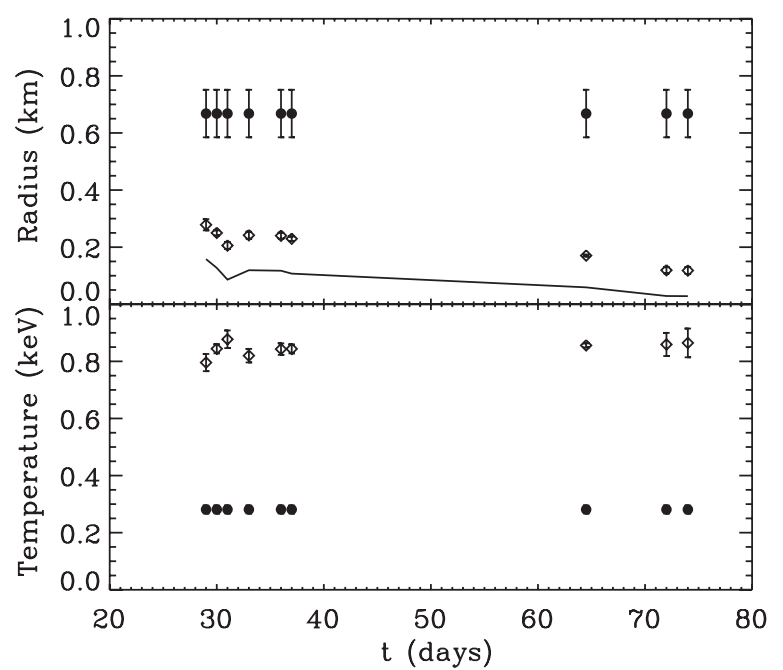

Figure 2. Time evolution of the temperature and emitting radius of the two BB components in the spectrum of SGR 0418+5729; a source distance of $2 \mathrm{kpc}$ is assumed. Diamonds refer to the hot and filled circles to the cold component. The solid line shows the ratio of the emitting areas, $A_{\text {hot }} / A_{\text {cold }}$. Time is counted in days from the outburst onset, on 2009 June 5 20:38:24.000 UTC (MJD 54987.862)

Within the magnetar model this is interpreted as being due to the sudden development of a twist in the external magnetic field which then progressively decays. The twist likely affects only a limited bundle of (closed) field lines and the charges flowing along the current-carrying bundle heat the surface layers as they impact upon the star. When the magnetosphere untwists, the size of the heated region decreases (Beloborodov 2009). This picture is compatible with the results we obtained for SGR $0418+5729$ with the $\mathrm{BB}+\mathrm{BB}$ model assuming that the heated region corresponds to the area emitting the hotter $\mathrm{BB}$. This is superimposed (or close) to a cooler, larger cap which is responsible for the emission of the softer $\mathrm{BB}$. It is interesting to note that the analysis of the pulse profiles during the first stages of the outburst supports this view. The double-peaked pulse profile of SGR $0418+5729$ suggests, in fact, that the surface thermal map of the star comprises two warm caps, only one of which was involved in the heating process (Esposito et al. 2010). The predicted characteristic time for the outburst evolution is $\approx 5\left(\Phi / 10^{9} \mathrm{~V}\right)^{-1}\left(B / 10^{14} \mathrm{G}\right) \Delta \phi\left(A / 10^{12} \mathrm{~cm}^{2}\right) \mathrm{yr}$, where $\Phi$ is the discharge voltage and $A$ is the area of the surface region involved by the twist (Beloborodov 2009). Taking $B \sim 5 \times 10^{12} \mathrm{G}, \Delta \phi \sim$ $0.4 \mathrm{rad}$, and $A \sim 10^{11} \mathrm{~cm}^{2}$, we get for the characteristic time $\approx 0.02\left(\Phi / 10^{9} \mathrm{~V}\right)^{-1} \mathrm{yr}$. A low discharge voltage, $\Phi \approx 10^{8} \mathrm{~V}$, is then required to obtain a decay time $\approx$ a few months. We warn that, as already noted by Esposito et al. (2010), the luminosity produced by Ohmic dissipation appears to be too low to reproduce that observed at the beginning of the outburst, $\sim 10^{34}(D / 2 \mathrm{kpc})^{2} \mathrm{erg} \mathrm{s}^{-1}$. However, if the twist affected a region different from a polar cap (e.g., a ring confined between two values $\theta_{1}$ and $\theta_{2}$ of the magnetic colatitude) the value of the luminosity can be higher. ${ }^{8}$ Alternatively, other heating mechanisms may be operating, e.g., the release of magnetic energy in the star outer layers (Lyubarsky et al. 2002).

As discussed in Section 2.3, the internal field of SGR $0418+5729$ can be still large enough to produce crustal displacements so that magnetic helicity is transferred to the external field, twisting up the magnetosphere. The appearance

\footnotetext{
8 We thank A. Beloborodov for bringing this point to our attention.
}

of a twist is usually accompanied by the formation of a highenergy spectral tail, due to resonant cyclotron up-scattering of thermal surface photons, which is, however, not unambiguously detected in SGR 0418+5729. In order to investigate the properties of resonant cyclotron scattering spectra in the low-field regime $\left(B_{p} \leqslant 5 \times 10^{13} \mathrm{G}\right)$, we run a series of three-dimensional Monte Carlo simulations, using the relativistic transport code of Nobili et al. (2008a, 2008b), to which we refer for all details. We considered four values of the (polar) surface field $\left(B_{p}=10^{12}, 5 \times 10^{12}, 10^{13}, 5 \times 10^{13} \mathrm{G}\right)$, two values of the seed photon temperature $(k T=0.3,0.9 \mathrm{keV})$, and several values of the twist angle ${ }^{9}$ in the range $0.1 \mathrm{rad}<\Delta \phi<1.2 \mathrm{rad}$. The electron temperature and bulk velocity were fixed to $k T_{\mathrm{el}}=10 \mathrm{keV}$ and $v / c=0.5$ in all cases. A different choice of these parameters produce similar results provided that the scattering particles are mildly relativistic, as indeed required to reproduce the observed 1-10 keV spectra of SGRs/AXPs (see, e.g., Nobili et al. 2008a, 2008b; Zane et al. 2009, and references therein). Results are summarized in Figure 3, which shows the photon index of the non-thermal tail (computed in the $6-8 \mathrm{keV}$ range) as a function of the twist angle for the different values of $B_{p}$. The (average) index (in the same energy range) of the blackbody spectrum is marked by a dashed horizontal line: when the photon index approaches the line the spectrum becomes indistinguishable from a blackbody and no tail is present. As can be seen, while for $k T=0.3 \mathrm{keV}$ a non-thermal tail below $10 \mathrm{keV}$ appears for all the values of the twist, unless $B_{p}=10^{12} \mathrm{G}$ and $\Delta \phi \lesssim 0.3 \mathrm{rad}$, the upscattering of seed photons associated with the hotter component only produces a tail if $\Delta \phi \gtrsim 0.5 \mathrm{rad}$ and $B_{p}>10^{12} \mathrm{G}$. We stress that here we are considering photon energies below $10 \mathrm{keV}$, so the lack of a non-thermal spectral component for $k T=0.9 \mathrm{keV}$ only reflects the fact that now resonant Comptonization tends to move photons at energies higher than $10 \mathrm{keV}$. A tail, in fact, may be present above $10 \mathrm{keV}$ also if it does not show up below $10 \mathrm{keV}$.

Although the resonant scattering spectrum produced by the reprocessing of soft photons coming from two NS surface regions at different temperatures is not exactly given by the superposition of the two individual models (see the discussion in Albano et al. 2010), we adopt this approach to get some insight into the spectral properties of SGR 0418+5729. If the observed spectrum of the source is best modeled in terms of the superposition of two blackbodies with $k T \sim 0.3,0.9 \mathrm{keV}$ the twist angle must be $\lesssim 0.5 \mathrm{rad}$ for $B_{p}>10^{12} \mathrm{G}$ to not produce a PL tail in the hot component (see Figure 3). This, however, is only a necessary condition because a PL tail may still appear in the cold component. The emergence of such a PL is related to the relative magnitude of the hot and cold components. The total spectrum resulting from the superposition of the models with $k T \sim 0.3$ and $0.9 \mathrm{keV}$ is shown in Figure 4 for $B_{p}=5 \times 10^{12} \mathrm{G}$ and two values of the ratio of the emitting areas, $A_{\text {cold }} / A_{\text {hot }}=15,30$, typical of those measured during the evolution of SGR $0418+5729$. We note that the largest area ratio $(=30)$ corresponds to the most unfavorable case: if the tail does not appear now it is not present for smaller values of the area ratio, when the cold component contributes less. As can be seen, the total spectrum is very close to the superposition of two blackbodies, with no high-energy tail. The same result holds for different values of the magnetic field, provided that $\Delta \phi \lesssim 0.5 \mathrm{rad}$, and for even larger values of the twist if the field is as low as $10^{12} \mathrm{G}$. We conclude that the strong evidence

\footnotetext{
9 Here the magnetosphere is assumed to be globally twisted.
} 

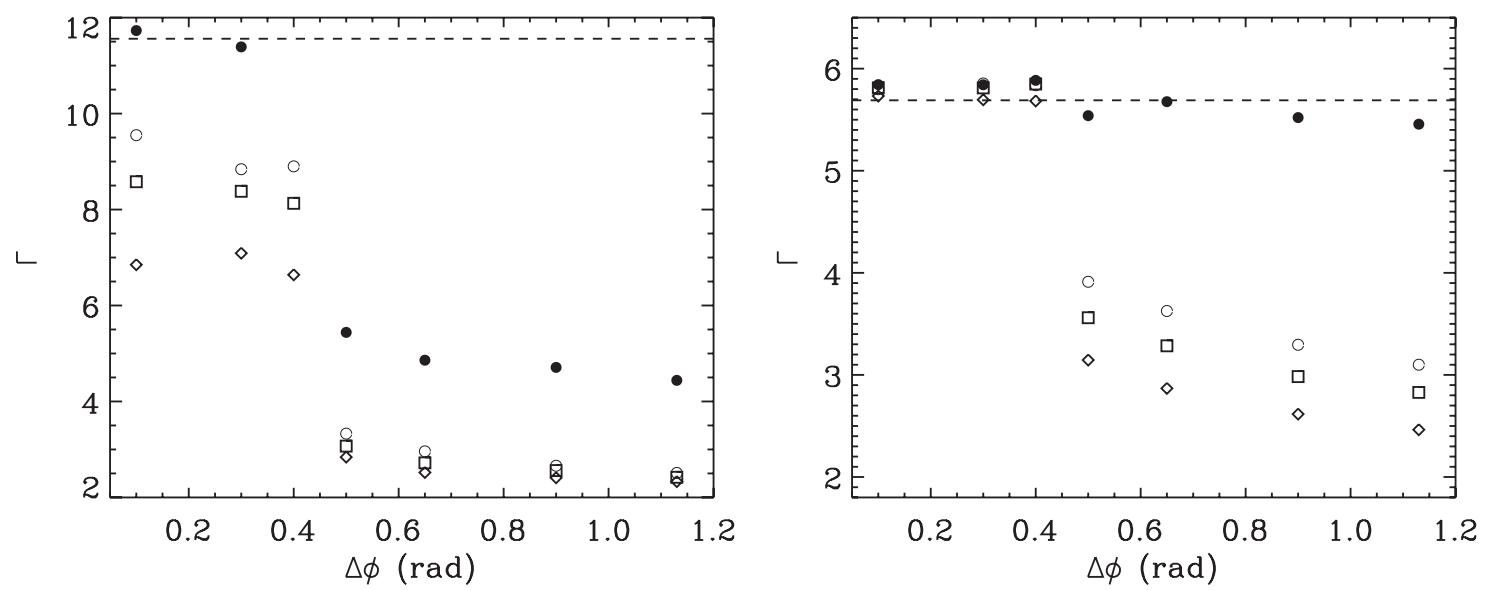

Figure 3. Left panel: the photon index $\Gamma$ vs. the twist angle $\Delta \phi$ for $B_{p}=10^{12} \mathrm{G}$ (filled circles), $5 \times 10^{12} \mathrm{G}$ (open circles), $10^{13} \mathrm{G}$ (squares), and $5 \times 10^{13} \mathrm{G}$ (diamonds); the seed photon temperatures is $k T=0.3 \mathrm{keV}$. The dashed horizontal line marks the value of the (average) index of the blackbody spectrum at the same temperature and in the same energy range (see the text). Right panel: same for $k T=0.9 \mathrm{keV}$.

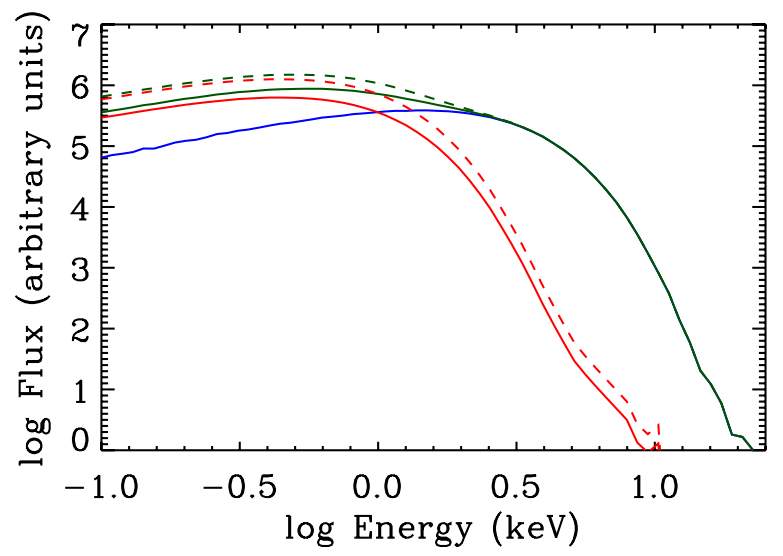

Figure 4. Spectra obtained from the superposition of two resonant scattering models with $k T=0.3,0.9 \mathrm{keV}, \Delta \phi=0.4 \mathrm{rad}, B_{p}=5 \times 10^{12} \mathrm{G}$. The cold component is shown in red, the hot in blue, and the sum in green. Solid (dashed) lines are for an emitting area ratio $A_{\text {cold }} / A_{\text {hot }}=15$ (30).

(A color version of this figure is available in the online journal.)

that SGR 0418+5729 exhibits an X-ray spectrum dominated by surface thermal emission below $10 \mathrm{keV}$ is not in contradiction with the predictions of RCS models.

\section{DISCUSSION}

SGR $0418+5729$ is the first "low-field" SGR/AXP ever discovered. Even if other sources of the same class with a relatively weak $B$-field were already known (e.g., 1E 2259+586, Gavriil \& Kaspi 2002), the case of SGR 0418+5729 is extreme and has stimulated considerable interest. With a surface dipole field $\lesssim 7.5 \times 10^{12}$ G, SGR $0418+5729$ seems to challenge an interpretation in terms of the "conventional" magnetar model, and not just because its magnetic field is below the quantum critical field, a quite irrelevant fact per se.

The present upper limit on the surface field in SGR 0418+5729 is based on the spin-down measure, which traces the dipole component. It is actually possible that the external magnetic field in NSs, and in SGRs/AXPs in particular, is more complex than a simple dipole. Higher order multipoles can substantially contribute to the field near the surface and, because they fall-off more rapidly with radius, induce negligible spin-down. SGR 0418+5729 may, then, possess a much higher surface $B$-field than indicated by its spin-down rate.
A high surface field in the form of multipolar components hints toward the presence a large internal field which can produce crustal motions and bursting activity, making SGR 0418+5729 not dissimilar (a part from the magnetic field topology) from other magnetar sources. It does not, however, explain the rotational properties of the source. With a dipole field below $10^{13} \mathrm{G}$, in fact, it would be impossible to slow down the star to the observed $9.1 \mathrm{~s}$ period in less than $\sim 24 \mathrm{Myr}$, a time much longer than the estimated age of other SGRs/AXPs, unless SGR $0418+5729$ had an exceptionally long period at birth. A possible solution was suggested by Alpar et al. (2011) who have shown that if SGR $0418+5729$ was born with a period $>70 \mathrm{~ms}$ and a low dipolar field $\left(B \approx 10^{12} \mathrm{G}\right)$, the torque exerted by a fallback disk can spin down the star to the present period in $\gtrsim 10^{5} \mathrm{yr}$, if $\dot{P}$ has to be below the observed upper limit. ${ }^{10}$ The survival of multipolar field components $\approx 100$ times stronger than the dipole over such a time span may, however, be an issue in the light of the known evolution of the dipolar field in magnetars (see Section 2.1).

In this paper, we explored a different possibility, i.e., that SGR $0418+5729$ is an old NS born with a super-strong magnetic field which experienced field decay over a time $\approx 10^{6}$ yr. Our results show that magneto-dipolar braking can effectively spin down the star to a period $\sim 10 \mathrm{~s}$ with $P \lesssim 10^{-15} \mathrm{~s} \mathrm{~s}^{-1}$ provided that the initial internal toroidal field is large enough, $B_{\text {tor }}(t=0) \gtrsim 10^{16} \mathrm{G}$. At the same time the initial external dipole field has to be $\lesssim(2-3) \times 10^{14} \mathrm{G}$ in order to prevent the NS from spinning down too fast. In particular, the model with $B_{\text {tor }}(t=0)=4 \times 10^{16} \mathrm{G}$ and $B_{p}(t=0)=2.5 \times 10^{14} \mathrm{G}$ gives $B_{p} \sim 2 \times 10^{12} \mathrm{G}, P \sim 9 \mathrm{~s}$, and $\dot{P} \sim 4 \times 10^{-16} \mathrm{~s} \mathrm{~s}^{-1}$ at an age of $\sim 1.5 \mathrm{Myr}$, in very good agreement with the current observational picture of SGR $0418+5729$. The predicted quiescent luminosity at the same age is $L_{X} \sim 10^{31} \mathrm{erg} \mathrm{s}^{-1}$, again in agreement with the current luminosity of the source, the faintest measured so far and possibly close to the quiescent value ( $L_{X} \sim 6 \times 10^{31} \mathrm{erg} \mathrm{s}^{-1}$ for a distance of $2 \mathrm{kpc}$; Rea et al. 2010). We note in this respect that the luminosity drops very quickly for ages $\gtrsim 10^{6} \mathrm{yr}$ (top left panel of Figure 1), so the present value could well be below $\sim 10^{31} \mathrm{erg} \mathrm{s}^{-1}$ if the age is only slightly above $1.5 \mathrm{Myr}$. A somehow longer age would have

\footnotetext{
10 A somehow similar scenario in which SGRs/AXPs are NSs with a low dipole field and super-strong multipolar components powered by accretion from a fallback disk was recently proposed by Trümper et al. (2010).
} 
negligible impact on the predicted $P, \dot{P}$, and $B_{p}$ which already reached a nearly constant value (see again Figure 1). We also note that the measured flux in the $\sim 0.5-10 \mathrm{keV}$ band may not be representative of the bolometric luminosity for an old object like SGR 0418+5729. If the NS surface temperature is $\lesssim 10^{6} \mathrm{~K}$, in fact, the quiescent thermal emission would be too soft (and absorbed) to be clearly detectable. It is then possible that the observed X-ray flux only constrains the "outburst" luminosity and the genuine quiescent emission may go undetected even if it is $\approx 10^{31} \mathrm{erg} \mathrm{s}^{-1}$.

Given the large internal toroidal field at birth, our fiducial model for SGR $0418+5729$ retains the capability to induce crustal fractures, and hence to produce bursts, even at quite late times. A comparison of our model with one of the cases investigated in detail by Perna \& Pons (2011) at a comparable age $(\sim 1.5 \mathrm{Myr})$ indicates that the recurrence time between bursting/outbursting events for an object like SGR 0418+5729 is a few tens of years. Crustal displacements are accompanied by a twisting up of the magnetosphere and the formation of a high-energy spectral tail, which is observed in most SGRs/ AXPs. We have shown, however, that a source with a spectral distribution consistent with a double blackbody, as follows from the analysis of a series of X-ray observations taken during the outburst decay of SGR $0418+5729$, can be modeled as well by a resonant cyclotron scattering model if the twist is moderate (twist angle $\lesssim 0.5 \mathrm{rad}$ ) and the surface field is low, $\lesssim 5 \times 10^{12} \mathrm{G}$, as predicted by our evolutionary calculations.

It has been already noticed that the SGRs/AXPs which exhibit the larger flux variations seem to be those with the lower dipole fields (Esposito et al. 2009). SGR 0418+5729 provides a further case for such a correlation. In the magnetar scenario the occurrence and energetics of outbursts are dictated by the internal field and the peak luminosity $L_{\max }$ depends on the (maximum) energy stored locally prior to the event. $L_{\max }$ is not very sensitive to the external dipole field. If the energy released in an event is roughly similar in all sources (as in the case that the mechanism is gated, e.g., by the crustal yield), the ratio between the peak and persistent fluxes has to be much higher in the less active, old objects than in active, young ones. This is because $L_{\text {max }}$ is similar for all of them, but the quiescent luminosity is much lower for old sources. In the latter, field decay had the time to reduce the dipole field to rather low values, so old, lowfield objects (but with sufficiently high internal fields) appear as transient sources. It is intriguing that SGR 0418+5729 is the source with the lowest field known and at the same time a most extreme transient, with a peak-to-persistent flux ratio of $\sim 1000$.

This research was partially funded through grants AAE I/088/06/0 (R.T.), AYA 2010-21097-C03-02, GVPROMETE02009-103 (J.P.) and AYA2009-07391, SGR2009-811, TW2010005 (N.R.). N.R. also acknowledges support from a
Ramon y Cajal Fellowship. P.E. acknowledges financial support from the Autonomous Region of Sardinia through a research grant under the program PO Sardegna FSE 2007-2013, L.R. 7/2007 "Promoting scientific research and innovation technology in Sardinia."

\section{REFERENCES}

Aguilera, D. N., Pons, J. A., \& Miralles, J. A. 2008, A\&A, 486, 255

Albano, A., Turolla, R., Israel, G. L., et al. 2010, ApJ, 722, 788

Alpar, M. A., Ertan, Ü., \& Çalişkan, Ş. 2011, ApJ, 732, L4

Beloborodov, A. M. 2009, ApJ, 703, 1044

Braithwaite, J., \& Spruit, H. C. 2006, A\&A, 450, 1097

Duncan, R. C., \& Thompson, C. 1992, ApJ, 392, 9

Esposito, P., Burgay, M., Possenti, A., et al. 2009, MNRAS, 399, L44

Esposito, P., Israel, G. L., Turolla, R., et al. 2010, MNRAS, 405, 1787

Esposito, P., Israel, G. L., Turolla, R., et al. 2011, MNRAS, in press (arXiv:1105.1323)

Gavriil, F. P., Gonzalez, M. E., Gotthelf, E. V., et al. 2008, Science, 319, 1802

Gavriil, F. P., \& Kaspi, V. M. 2002, ApJ, 567, 1067

Geppert, U., Küker, M., \& Page, D. 2004, A\&A, 426, 267

Geppert, U., Küker, M., \& Page, D. 2006, A\&A, 457, 937

Glampedakis, K., Jones, D. I., \& Samuelsson, L. 2011, MNRAS, 413, 2021

Goldreich, P., \& Reisenegger, A. 1992, ApJ, 395, 250

Gotthelf, E. V., \& Halpern, J. P. 2007, Ap\&SS, 308, 79

Kaspi, V. M. 2010, Proc. Natl Acad. Sci., 107, 7147

Kumar, H. S., \& Safi-Harb, S. 2008, ApJ, 678, L43

Levin, L., Bailes, M., Bates, S., et al. 2010, ApJ, 721, L33

Lyubarsky, Yu., Eichler, D., \& Thompson, C. 2002, ApJ, 580, L69

Mereghetti, S. 2008, A\&AR, 15, 225

Nobili, L., Turolla, R., \& Zane, S. 2008a, MNRAS, 386, 1527

Nobili, L., Turolla, R., \& Zane, S. 2008b, MNRAS, 389, 989

Page, D., Lattimer, J. M., Prakash, M., \& Steiner, A. W. 2004, ApJS, 155, 623

Page, D., Prakash, M., Lattimer, J. M., \& Steiner, A. W. 2011, Phys. Rev. Lett., 106, 081101

Pérez-Azorín, J. F., Miralles, J. A., \& Pons, J. A. 2006, A\&A, 451, 1009

Perna, R., \& Pons, J. A. 2011, ApJ, 727, L51

Pons, J. A., \& Geppert, U. 2007, A\&A, 470, 303

Pons, J. A., Miralles, J. A., \& Geppert, U. 2009, A\&A, 496, 207

Rea, N., \& Esposito, P. 2011, in Proc. Sant Cugat Forum on Astrophysics, Highenergy Emission from Pulsars and Their Systems, ed. N. Rea \& D. F. Torres (Berlin: Springer), 247

Rea, N., Esposito, P., Turolla, R., et al. 2010, Science, 330, 944

Rea, N., Zane, S., Turolla, R., \& Lyutikov, M. 2008, ApJ, 686, 1245

Shternin, P. S., Yakovlev, D. G., Heinke, C. O., Ho, W. C. G., \& Patnaude, D. J. 2011, MNRAS, 412, L108

Thompson, C., \& Duncan, R. C. 1993, ApJ, 408, 194

Thompson, C., \& Duncan, R. C. 1995, MNRAS, 275, 255

Thompson, C., Lyutikov, M., \& Kulkarni, S. R. 2002, ApJ, 274, 332

Tiengo, A., Esposito, P., \& Mereghetti, S. 2008, ApJ, 680, L183

Trümper, J. E., Zezas, A., Ertan, Ü., \& Kylafis, N. D. 2010, A\&A, 518, 46

Turolla, R. 2009, in Neutron Stars and Pulsars, ed. W. Becker (Astrophysics and Space Science Library; Berlin: Springer), 357

van der Horst, A. J., Connaughton, V., Kouveliotou, C., et al. 2010, ApJ, 711, L1

Woods, P. M., \& Thompson, C. 2006, in Compact Stellar X-ray Sources, ed. W. Lewin \& M. van der Klis (Cambridge: Cambridge Univ. Press), 547

Zane, S., Rea, N., Turolla, R., \& Nobili, L. 2009, MNRAS, 398, 1403

Zane, S., \& Turolla, R. 2006, MNRAS, 366, 727 